\section{Möglichkeiten und Grenzen der Beteiligung an MVZ auf Gründer- und Trägerebene}

\section{Einleitung}

Die ambulante vertragsärztliche Versorgung ist nach wie vor geprägt durch den niedergelassenen Vertragsarzt, der seine Tätigkeit in "freier Praxis" auszuüben hat (vgl. § 98 Abs. 2 Nr. 13 SGB V, § 32 Abs. 1 Ärzte-ZV). Die vertragsärztliche Zulassung ist damit weiterhin Ausgangspunkt jeder Gründungsentscheidung im Vertragsarztrecht. Das Leitbild des zugelassenen Vertragsarztes ist jedoch durch die Einführung von Medizinischen Versorgungszentren (MVZ), die auch durch nicht an der vertragsärztlichen Versorgung teilnehmende Leistungserbringer und in anderen Rechtsformen als Berufsausübungsgemeinschaften (BAG) nach § 33 Abs. 2 und 3 Ärzte-ZV gegründet werden können (vgl. §95 Abs. 1a S. 1 SGB V), eingeschränkt worden. Soweit MVZ jedoch nicht durch Vertragsärzte gegründet werden, bei denen bereits das ärztliche Berufsrecht die ausschließliche Beteiligung berechtigter Gründer garantiert ${ }^{1}$, stellt sich insbesondere für Krankenhäuser nach $\S \S 107,108$ SGB V und für Erbringer nichtärztlicher Dialyseleistungen nach § 126 Absatz 3 SGB V die Frage, ob neben deren Zulassung auch deren innere Struktur dafür maßgeblich ist, ob diese zur Gründung von MVZ berechtigt sind und welche Rechte diesen gegenüber den MVZTrägergesellschaften zustehen.

Der Bundesgerichtshof (BGH) hatte mit Urteil vom 19.08.2020² darüber zu entscheiden, ob die Beteiligung von Apothekern an einer MVZ-Trägergesellschaft im Rahmen einer sog. Strohmannkonstruktion als Abrechnungsbetrug zulasten der Kassenärztlichen Vereinigung (KV) zu bewerten ist. In dem der Entscheidung zu Grunde liegenden Sachverhalt war ein Apotheker verdeckt an der Trägergesellschaft eines MVZ

1 Auch dann, wenn Vertragsärzte ein MVZ in der Rechtsform der $\mathrm{GmbH}$ betreiben, sind nach Auffassung des BSG besondere Anforderungen an die innere Struktur der Gesellschaft zu stellen, die sich aus den Vorgaben der Ärztegesellschaft in § 23a Musterberufsordnung ergeben, vgl. BSG, Urt. v. 29.11.2017, Az.: B 6 KA 31/16 R.

2 BGH, Urt. v. 19.08.2020, Az.: 5 StR 558/19. beteiligt. Unter einem Strohmann versteht man ein Rechtssubjekt, das bei Geschäften oder sonstigen Rechtshandlungen für eine andere Person (Hintermann) auftritt, die selbst nicht in Erscheinung treten will, kann oder darf. Die Entscheidung des BGH gibt Anlass dazu, die Möglichkeiten und Grenzen der Beteiligung an MVZ auf Trägerund Gründerebene genauer zu beleuchten, denn unzulässige Vertragsgestaltungen sind nicht nur vertragsarztrechtlich und zivilrechtlich unzulässig, sondern können auch strafrechtliche Konsequenzen nach sich ziehen.

\section{Die rechtliche Organisation des MVZ im Überblick}

Für das Verständnis ist in diesem Zusammenhang zunächst bedeutsam, dass bei MVZ, anders als bei BAG, grundlegend zwischen der Gründerebene, der Trägerebene und der Betriebs- bzw. Zulassungsebene zu unterscheiden ist. Diese drei Ebenen sind durch folgende Handlungsbereiche gekennzeichnet: Der Gründer stellt die rechtliche Einheit dar, die die Gesellschaft bzw. rechtliche Organisationsform gründet (Gründerebene); diese wiederum betreibt dann das MVZ (Trägerebene). Die Betriebs- bzw. Zulassungsebene beschreibt die Organisation des MVZ und die beim Betrieb zu beachtenden rechtlichen Anforderungen. ${ }^{3}$ Diese drei Ebenen sind grundsätzlich unabhängig voneinander zu beurteilen; sie stehen dabei jedoch miteinander in Wechselwirkung.

Das MVZ ist eine besondere Organisationsund Kooperationsform im Rahmen der vertragsärztlichen Versorgung. ${ }^{4}$ Es selbst ist Träger der Zulassung und Adressat der Anstellungsgenehmigung im Rahmen des Vertragsarztrechts, nicht jedoch die jeweilige Trägergesellschaft, die das MVZ betreibt. ${ }^{5}$

3 Kremer/Wittmann, Vertragsärztliche Zulassungsverfahren, 2018, Rn. 838.

4 BSG, Urt. v. 19.02.2014, Az.: B 6 KA 8/13R, SozR 4-2500 § $85 \mathrm{Nr} 80$, Rn. 35.

5 Rademacker in: Kasseler Kommentar zum Sozialrecht, 111. EL September 2020, SGB V § 95 Rn. 93.

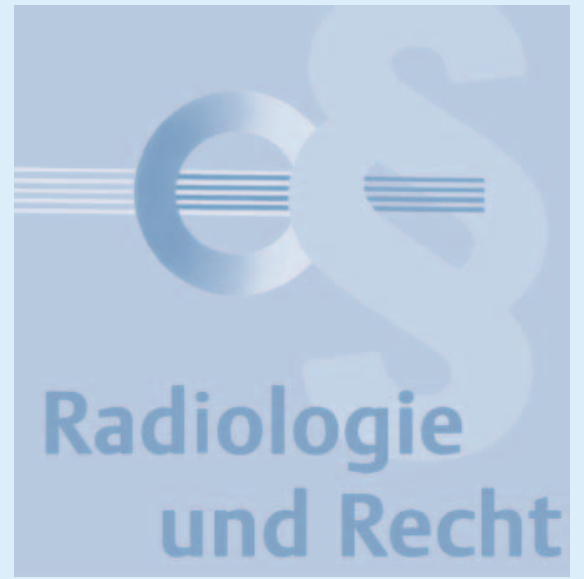

\section{Historische Entwicklung}

Die Kooperationsform des MVZ hat sich seit seiner Einführung über die Jahre hinweg erheblich gewandelt. Während zum Zeitpunkt seiner Einführung im Jahre 2003 durch das GKV-Modernisierungsgesetz ${ }^{6}$ neben Vertragsärzten noch ein sehr weiter Kreis von Leistungserbringern gründungsberechtigt, die zulässigen Organisationsformen kaum eingeschränkt und eine fachübergreifende Kooperation vorgeschrieben war, modifizierte der Gesetzgeber das Konzept des MVZ bis heute immer wieder. Insbesondere der Gründerkreis wurde schrittweise eingeschränkt, da nach der Begründung des Gesetzgebers die Gefahr bestehe, dass sich Dritte an den MVZ beteiligen, die ausschließlich wirtschaftliche Interessen mit dem Betrieb eines MVZ verfolgen würden. Insbesondere wird bei Investoren die Gefahr gesehen, „dass medizinische Entscheidungen von Kapitalinteressen beeinflusst werden. “7

Mit dem GKV-VStG ${ }^{8}$ aus dem Jahre 2011 wurden daher sowohl der Gründerkreis als auch die rechtlich zulässigen Organisationsformen weiter eingeschränkt. Während man mit dem GKV-VSG ${ }^{9}$ im Jahr 2015 das Erfordernis des fachübergreifenden

6 GKV-Modernisierungsgesetz (GMG) vom 14.11.2003, BGBI. I, 2003, 2190, in Kraft getreten am: 01.01.2004

7 BT-Drs. 17/6906 S. 70.

8 GKV-Versorgungsstrukturgesetz (GKV-VStG) vom 22.11.2011, BGBI. I, 2011, 2983, in Kraft getreten am: 01.01.2012.

9 GKV-Versorgungsstärkungsgesetz (GKV-VSG) vom 16.07.2015, BGBI. I, 2015, 1211, in Kraft getreten am: 23.07.2015. 
Charakters aufhob, beschränkte der Gesetzgeber im Jahr 2019 durch das TSVG ${ }^{10}$ die Befugnis zur Gründung von MVZ durch Erbringer nichtärztlicher Dialyseleistungen sowie durch Krankenhäuser noch weitgehender.

\section{Wesentliche Unterschiede der Gründer in Bezug auf die MVZ-Gründungsbefugnis}

MVZ können gemäß § 95 Abs. 1a SGB V unter anderem von zugelassenen Ärzten, zugelassenen Krankenhäusern und Erbringern nichtärztlicher Dialyseleistungen nach §126 Abs. 3 SGB V (im Folgenden abgekürzt: näD) gegründet werden. Die nachfolgende Darstellung beschränkt sich auf diese vorgenannten berechtigten Gründer, weil diese neben Vertragsärzten den größten Anteil der MVZ-Gründer darstellen. ${ }^{11}$

Zugelassene Ärzte sind nach §95 Abs. 1a SGB V - im Gegensatz zu zugelassenen Krankenhäusern und näD - ohne Einschränkung zur Gründung von MVZ berechtigt. Hierbei handelt es sich um natürliche Personen, welche ohnehin schon aufgrund ihrer Zulassung an der vertragsärztlichen Versorgung teilnehmen.

NäD und zugelassene Krankenhäuser erhalten erst durch die Gründung von MVZ Zugang zur vertragsärztlichen Versorgung, da sie primär im Bereich der Hilfsmittelversorgung bzw. im stationären Bereich tätig sind. Aufgrund ihrer Organisationsstruktur als juristische Personen besteht grundsätzlich die Möglichkeit, dass sich auf der Gründerebene natürliche oder juristische Personen, welche selbst nicht zur Gründung von MVZ nach § 95 Abs. 1a SGB V berechtigt sind, an diesen beteiligen. Dies ist bei zugelassenen Ärzten - als natürliche Personen - der Natur der Sache nach ausgeschlossen. Vertragsärzte sind auch innerhalb von

10 Terminservice- und Versorgungsgesetz (TSVG) vom 6.05.2019, BGBI. I, 2019, 646, in Kraft getreten am: 11.05.2019.

11 Vgl. Kassenärztliche Bundesvereinigung, Medizinische Versorgungszentren aktuell, Statistische Informationen zum Stichtag 31.12.2019 (Stand:16.11.2020), abrufbar unter: https://www.kbv.de/media/sp/ mvz-aktuell.pdf
MVZ-Strukturen an das ärztliche Berufsrecht gebunden. Dieses findet aber weder auf das MVZ selbst noch auf die anderen genannten Leistungserbringer als berechtigte Gründer Anwendung. ${ }^{12}$

Im Rahmen des TSVG im Jahre 2019 wurde zum einen die Gründungsbefugnis der näD beschränkt. Diese sind nach $\S 95$ Abs. 1a S. 2 HS 1 SGB V nur noch zur Gründung von sog. „fachbezogenen“ MVZ berechtigt. Wie weit oder eng dieser Fachbezug zu verstehen ist, ist im Einzelfall streitig. ${ }^{13}$ Diese Beschränkung der Gründungsbefugnis stellt gleichzeitig durch § 95 Abs. 6 S. 3 SGB $\checkmark$ eine Beziehung zur Betriebsebene her, wenn ein nach dem Inkrafttreten des TSVG gegründetes MVZ sein Versorgungsangebot erweitert. Dieser regelt:

„Einem medizinischen Versorgungszentrum ist die Zulassung auch dann zu entziehen, wenn die Gründungsvoraussetzungen des $A b$ satzes 1a Satz 1 bis 3 länger als sechs Monate nicht mehr vorliegen."

Bei zugelassenen Krankenhäusern handelt es sich um solche nach $\S \S 107,108$ SGB V, also Einrichtungen, die den Krankenhausbegriff nach § 107 SGB V erfüllen und die über eine Zulassung nach § 108 SGB V verfügen. Dies sind solche Krankenhäuser, die nach landesrechtlichen Vorschriften als Hochschulklinik anerkannt sind (Nr. 1), Plankrankenhäuser (Nr. 2) und Krankenhäuser, die einen Versorgungsvertrag mit den Landesverbänden der Krankenkassen und den Verbänden der Ersatzkassen abgeschlossen haben (Nr. 3). Ihre Gründungsbefugnis wurde nun ausschließlich hinsichtlich zahnärztlicher MVZ durch das TSVG beschränkt. Ein zahnärztliches MVZ kann von diesen seither nur noch dann gegründet werden, wenn dies aus Versorgungsgesichtspunkten (Versorgungsanteil und Versorgungsgrad) erforderlich ist (§95 Abs. 1b SGB V).

12 BSG, Urt. v. 09.02.2011, Az.: B 6 KA 12/10 R. 13 Vgl. hierzu Rademacker in: Kasseler Kommentar zum Sozialrecht, 111. EL September 2020 , SGB V § 95 Rn. 65; Bördner KrV 2019, 193 , 198.

\section{Urteil des BGH vom 19.08.2020}

In dem Urteil des $\mathrm{BGH}^{14}$ strebte ein Apotheker (Angeklagter Z), der seit der Beschränkung der Gründungsbefugnis im Jahr 2012 nicht mehr zur Gründung von MVZ berechtigt war, zur Steigerung des Absatzes der von ihm vertriebenen Arzneimittel die Beteiligung an einem MVZ an. Gemeinsam mit seinem Rechtsberater entwickelte er die Idee, die gesetzlichen Vorgaben dadurch zu umgehen, dass er über einen Arzt als „Strohmann“ Anteile an einem MVZ erwirbt. Ein niedergelassener Vertragsarzt, der Angeklagte D, erklärte sich bereit, „auf dem Papier“ die Funktion eines Gesellschafters einer MVZ-Trägergesellschaft des Angeklagten $\mathrm{F}$ zu übernehmen. Der Angeklagte $D$ sollte seine Gesellschafterrechte ausschließlich gemäß den Anweisungen des Angeklagten $Z$ ausüben. $Z$ finanzierte den Erwerb der Geschäftsanteile und stellte D von sämtlichen mit der Beteiligung verbundenen unternehmerischen Risiken frei. Hierfür erhielt $D$ eine Vergütung in unbekannter Höhe. Er verpflichtete sich, an Z die Gewinne aus dem Betrieb des MVZ abzuführen. Nach dem Erwerb der Gesellschaftsanteile durch D kam es zu deutlichen Umsatzsteigerungen in der Apotheke des Z, die insbesondere auf Verordnungen von Ärzten dieses MVZ beruhten.

Der BGH befand den Angeklagten Z des banden- und gewerbsmäßigen Betruges zulasten der Kassenärztlichen Vereinigung für schuldig, da ein Fall des Gestaltungsmissbrauchs anzunehmen sei. Die vorgegebenen formalen Verhältnisse entsprächen nicht den tatsächlichen Gegebenheiten und seien daher gänzlich nicht abrechenbar. ${ }^{15}$ Nach den Feststellungen des BGH war der Angeklagte D ein bloßer „Strohmann“, während tatsächlich der Angeklagte $\mathbf{Z}$ die Funktion eines Gesellschafters der MVZ-Trägergesellschaft innehatte. Der KV sei insoweit ein Schaden entstanden, obwohl tatsächlich Patienten behandelt wurden. 
Die vertragsarztrechtlich unzulässige Gestaltung fand in dem dem Urteil zu Grunde liegenden Sachverhalt auf der Trägerebene des MVZ statt.

\section{Möglichkeiten und Grenzen der Beteiligung auf Träger- und Gründerebene}

Das BGH-Urteil vom 19.08.2020 illustriert die Grenzen, die den Möglichkeiten der Beteiligung auf Trägerebene eines MVZ gesetzt sind. Nur berechtigte Gründer nach §95 Abs. 1a SGB V dürfen Gesellschafter der Trägergesellschaft eines MVZ sein. Nur dann liegt eine formal zulässige Gestaltung vor, welche es ermöglicht, die im MVZ erbrachten Leistungen auch gegenüber der KV abzurechnen.

Darüber hinaus ist einem MVZ gemäß § 95 Abs. 6 S. 3 SGB V allerdings auch dann die Zulassung zu entziehen, wenn die Gründungsvoraussetzungen länger als sechs Monate nicht mehr vorliegen.

Zum Teil wird bereits die Übertragung von Anteilen an einer MVZ-Trägergesellschaft an einem nichtberechtigten Gründer als ein Verstoß gegen ein gesetzliches Verbot gemäß § 134 BGB i. V. m. §95 Abs. 1a SGB $V$ und damit als unwirksam angesehen. ${ }^{16}$ Eine solche Auslegung des Gesetzes ist jedoch fragwürdig: Ein Verbot ist dadurch gekennzeichnet, dass nach Auslegung der Norm ein bestimmtes Rechtsgeschäft schlechterdings nicht vorgenommen werden soll. ${ }^{17}$ Näher liegt es jedoch, dass sich aus $\S 95$ Abs. 6 S. 3 SGB V „ein anderes ergibt“ im Sinne des § 134 BGB, sodass das Geschäft nicht nichtig ist. Denn das Sozialrecht sanktioniert den Verstoß gegen die Gründungsvoraussetzungen gesondert. Folge hiervon ist lediglich die Entziehung der Zulassung und das auch erst dann, wenn die Gründungsvoraussetzungen sechs Monate nicht mehr vorliegen (vgl.

16 Vgl. LG Köln, Urt. v. 01.12.2016 Az.: 5 O 236/15, MedR 2017, 488.

17 Armbrüster in: Münchener Kommentar zum BGB, 8. Aufl. 2018, BGB § 134 Rn. 41, 42. den obigen Wortlaut). ${ }^{18}$ Insoweit bestimmt das Sozialrecht selbst eine abweichende Folge, „ein anderes“, auf Grund dieses Verstoßes. Dies soll jedoch an dieser Stelle nicht weiter vertieft werden.

\section{Grenzen der Beteiligung an berechtigten Gründern}

Soweit die Beteiligung von nichtberechtigten Gründern an einem berechtigten Gründer, wie einem zugelassenen Krankenhaus oder einem näD, im Sinne des §95 Abs. 1a S. 1 SGB V in Rede steht, ist diese Lösung jedoch weniger eindeutig und wurde durch die Rechtsprechung bisher noch nicht entschieden. Wie mit dieser Situation umgegangen werden könnte, wird im Folgenden aufgezeigt:

Zum Teil wird die Beteiligung an berechtigten Gründern kritisiert oder unter Berücksichtigung bestimmter Voraussetzungen für unzulässig erachtet. Unter Hinweis auf den oben dargelegten Willen des Gesetzgebers im Jahre 2011 wird teilweise allein nach dem Umfang der Beteiligung differenziert. Eine Mehrheitsbeteiligung soll ohne weiteres zulässig sein. Eine Minderheitsbeteiligung sei hingegen dann unzulässig, wenn diese lediglich als Vehikel zum Betrieb eines MVZ dient und der Betrieb sowie das unternehmerische Risiko aufgrund der konkreten vertraglichen Gestaltung faktisch allein beim Investor liegt. Dies sei immer eine Frage des Einzelfalls. ${ }^{19}$

Zum Teil wird aber auch nicht nach dem Umfang der Beteiligung, sondern ausschließlich nach Wertungsgesichtspunkten differenziert. Eine Beteiligung sei dann unzulässig, wenn der berechtigte Gründer ein bloßer Strohmann des Hintermanns sei. Dies sei danach zu werten, ob der berechtigte Gründer zur Verschleierung der tatsächlichen Verhältnisse als Antragsteller vorgeschoben wird, das MVZ bei wertender Betrachtung aber von einem anderen betrieben wird. Dies sei nur dann der Fall, wenn eine genaue Analyse der Innenbeziehungen zeige, dass der Antragsteller ohne

18 Ebenso für §33 Abs. 2 Ärzte-ZV: Vossler in: BeckOGK, Stand: 1.9.2020, BGB § 134 Rn. 187; Nassall in: Herberger/Martinek/Rüßmann/Weth/Würdinger, jurisPK-BGB, Stand: 18.05.2020, § 134 BGB Rn. 110.

19 Bäune/Dahm/Flasbarth, MedR 2012, 77, 79. eigene unternehmerische Tätigkeit nur als Marionette des Hintermanns am Geschäftsleben teilnimmt. ${ }^{20}$

Einer derartigen Auslegung des Gesetzes kann jedoch entgegengehalten werden, dass sie grundlegenden verfassungsrechtlichen Anforderungen widerspricht und sie jedenfalls seit Inkrafttreten des TSVG nicht mit dem ausgeformten Willen des Gesetzgebers in Einklang zu bringen ist. Probleme ergeben sich insbesondere mit Blick auf den Vorbehalt des Gesetzes.

\section{Vorbehalt des Gesetzes}

Ein jeder Rechtseingriff bedarf einer rechtlichen Grundlage, Art. 20 Abs. 3 Hs. 2 GG. Aus dem Vorbehalt des Gesetzes folgt für die vollziehende Gewalt das verfassungsrechtliche Verbot, ohne wirksam gewordene gesetzliche Grundlage tätig zu werden. ${ }^{21}$ Dieser Vorbehalt des Gesetzes gilt einfachgesetzlich auch im Sozialrecht gemäß § 31 SGB I. ${ }^{22}$

Soweit man also eine Beteiligung an einem berechtigten Gründer als unzulässig erachtet, was die Entziehung oder Versagung der Zulassung eines MVZ zur Folge hätte, bedarf es hierfür einer rechtlichen Grundlage. Im Hinblick auf das Zulassungsrecht kommt eine teleologische Auslegung des §95 Abs. 1a S. 1 SGB V in Betracht - eine Auslegung anhand des Sinns und Zwecks der Regelung. Zu klären ist, ob §95 Abs. 1a SGB V eine ausreichende gesetzliche Grundlage dafür bietet, die Zulassung zu entziehen, soweit sich nicht berechtigte Gründer an berechtigen Gründern beteiligen. Angesichts der Klarheit des Wortlauts des §95 Abs. 1a $S G B V$ ist eine derartige Auslegung eher nicht anzunehmen. Diese Vorschrift bestimmt eindeutig, dass näD oder zugelassene Krankenhäuser zur Gründung von MVZ berechtigt sind. Eine Anreicherung des Gesetzes mit derart weitreichenden Anforderungen oder auch Billigkeitserwägungen findet keine Wortlautanknüpfung.

20 Kremer/Wittmann, a. a. O. (Fn. 3), Rn. 869.

21 Sachs in: Sachs, Grundgesetzkommentar, 8. Aufl. 2018, GG Art. 20 Rn. 113.

22 Spellbrink in: Kasseler Kommentar zum Sozialrecht, 110. EL Juli 2020, SGB I § 31 Rn. 11; Mrozynski, SGB I, 5. Aufl. 2014, §31, Rn. 16. 
Selbst wenn man also aufgrund der Gesetzesbegründung einen Willen des Gesetzgebers bejaht, die Berechtigung zur Gründung von MVZ zu begrenzen, muss dieser hierfür selbst eine rechtliche Grundlage schaffen. Grundrechtswesentliche Entscheidungen sind durch den Gesetzgeber und nicht durch die Verwaltung zu treffen (Wesentlichkeitstheorie). ${ }^{23}$ Die Zulassungsentziehung als erheblicher Eingriff in die Berufsfreiheit aus Art. 12 Abs. 1 GG stellt eine solche wesentliche Entscheidung dar.

Zudem liegt auch kein unbestimmter Rechtsbegriff - also ein Begriff, der nicht aus sich heraus verständlich ist, sondern einen weiten Spielraum zur Interpretation zulässt - vor, welcher zumindest den Anwendungsbereich der Lehre vom Beurteilungsspielraum eröffnen würde; was wiederum unter der Berücksichtigung von Art. 19 Abs. 4 GG nur eingeschränkt zulässig ist. ${ }^{24}$ Auch eine richterliche Rechtsfortbildung scheidet dann aus, wenn sich der Richter über den klaren Wortlaut des Gesetzes hinwegsetzen würde und dadurch Rechtspositionen verkürzt werden. ${ }^{25}$

Wie bereits dargelegt lässt der aktuelle Wortlaut des §95 Abs. 1a S. 1 SGB V keinen Spielraum für zusätzliche Anforderungen an die gesellschaftsrechtliche Struktur der berechtigten Gründer. Dieser bestimmt (Hervorhebung durch die Verfasser):

„Medizinische Versorgungszentren können von zugelassenen Ärzten, von zugelassenen Krankenhäusern, von Erbringern nichtärztlicher Dialyseleistungen nach §126 Absatz 3, von anerkannten Praxisnetzen nach §87b Absatz 2 Satz 3, von gemeinnützigen Trägern, die aufgrund von Zulassung oder Ermächtigung an der vertragsärztlichen Versorgung teilnehmen, oder von Kommunen gegründet werden."

\section{Auslegung nach dem Willen des Gesetzgebers}

Das mit Erlass des GKV-VStG von 2011 erklärte Ziel, die ambulante Versorgung von

23 BVerfG, Beschl. v. 07.03.2017, Az.: 1 BvR 1314, 1630/12, 1694/13, 1874/13, m.w. N.

24 Sachs, a. a. O., Rn. 115.

25 Jachmann-Michel in: Maunz/Dürig, Grundgesetz Kommentar, 90. EL Februar 2020, GG Art. 95 Rn. 16.
Investoren freizuhalten, kann daher als nicht konsequent umgesetzt bezeichnet werden. ${ }^{26}$ Denn schon im Rahmen des Gesetzgebungsverfahrens im Jahre 2011 hatte der Freistaat Bayern gefordert, Regelungen aufzunehmen, welche verhindern, dass ein MVZ-Gründer eine marktbeherrschende Stellung erlangt. ${ }^{27}$ Stattdessen nahm man im Laufe des Gesetzgebungsverfahrens zusätzlich die näD in den Kreis der berechtigten Gründer auf.

Selbst wenn man einen solchen Willen des Gesetzgebers annimmt, hat sich dieser mittlerweile darauf hin geformt bzw. beschränkt, die Investorentätigkeit bei der Gründung von MVZ allenfalls zu lenken. Der Gesetzgeber weiß um die komplexen Beteiligungsstrukturen, welche teilweise im Kontext von MVZ Gründungen bestehen. ${ }^{28}$

Vom Bundesrat als problematisch vorgetragen wurde insbesondere:

„In immer mehr Bereichen der ambulanten ärztlichen Versorgung bilden sich konzernartige Strukturen aus, oft in der Hand renditeorientierter Unternehmen. In manchen Regionen, besonders in Ballungsräumen, sind alle oder ein Großteil der Arztsitze einer Fachgruppe in der Hand desselben Konzerns. Es besteht die Gefahr der Monopolisierung und damit der Verschlechterung der Patientenversorgung. "29

Der Gesetzgeber begrenzte jedoch im Zuge des TSVG sodann lediglich formal die Gründungsbefugnis der näD und zugelassenen Krankenhäuser und zwar vor dem erklärten Ziel, den

„Einfluss von Kapitalinvestoren ohne medizinisch-fachlichen Bezug zur vertragsärztlichen Versorgung auf die Versorgungsstrukturen zu begrenzen“30.

Eingedenk dessen verzichtete der Gesetzgeber also darauf, an die inneren gesellschaftsrechtlichen Strukturen der berechtigten

26 Möller/Dahm/Remplik in: Ratzel/Luxenburger, Handbuch des Medizinrechts, 3. Aufl. 2015, 9. Kapitel, Rn. 99.

27 BR Drs. 456/2/11, S. 1.

28 Vgl. BT-Drs. 19/8351, S. 189.

29 BT-Drs. 19/6337 S. $177 \mathrm{ff.}$

30 BT-Drs. 19/6337 S. 58.
Gründer weitergehende Anforderungen zu stellen. Vielmehr übte er seine Einschätzungsprärogative zum Beispiel im Bereich von zahnärztlichen MVZ dahingehend aus, eine Gründung eines MVZ vom Versorgungsanteil und Versorgungsgrad im Planbereichs des jeweiligen Krankenhauses abhängig zu machen. Der Gesetzgeber beschränkt sich somit auf eine marktlenkende Funktion unter besonderer Berücksichtigung von Versorgungsgesichtspunkten.

Der Gesetzgeber hat sich nach alledem nicht generell gegen Investorentätigkeit im Rahmen der Gründung von MVZ gewandt, sondern als Ziele die Aufrechterhaltung der freien Arztwahl durch Verhinderung der Monopolisierung, die Begrenzung der ärztlichen Versorgung auf Ballungsgebiete bzw. die Aufrechterhaltung der Versorgung im ländlichen Bereich und die allgemeine Sicherstellung einer qualitativ hochwertigen Patientenversorgung durch die Begrenzung des Einflusses von Kapitalinteressen auf ärztliche Entscheidungen verfolgt.

Angesichts des jedenfalls nach Erlass des TSVG eindeutig geformten und durch die Überarbeitung des $§ 95$ Abs. 1a, 1b SGB V zum Ausdruck gekommenen Willen des Gesetzgebers, besteht für eine weitergehende teleologische Auslegung des §95 Abs. 1a S. 1 SGB V wenig Raum. Soweit der Gesetzgeber eine weitergehende Beschränkung für erforderlich halten sollte, müsste dies zukünftig durch weitere Reformvorhaben geschehen.

\section{Zusammenfassung und Ausblick}

Zusammenfassend lässt sich festhalten, dass den Beteiligungsmöglichkeiten an MVZ auf Ebene der Trägergesellschaft erhebliche Grenzen gesetzt sind. Diese wurden durch die Rechtsprechung hinreichend konturiert und sind auch im Gesetz niedergelegt. Demgegenüber steht jedoch eine weitreichende Beteiligungsmöglichkeit an MVZ auf der Gründerebene, insbesondere bei zugelassenen Krankenhäusern und näD. Zwar wurde die Gründungsbefugnis von MVZ im Verlaufe der letzten Jahre immer weiter beschränkt. Hieraus lassen sich jedoch keine rechtlich verbindlichen Schlüsse auf weitergehende 
Anforderungen an die innere gesellschaftsrechtliche Struktur von berechtigten Gründern ziehen. Es bleibt daher abzuwarten, ob die derzeitigen Beschränkungen der Gründungsbefugnis das Ende dieser Entwicklung darstellen.

Der Bundesrat schlug im Rahmen des Gesetzgebungsverfahrens des TSVG zur weitergehenden Beschränkung der Gründungsbefugnis von Krankenhäusern die Aufnahme eines räumlich-regionalen Bezuges zum Krankenhausstandort, einen fachlichen Bezug des Krankenhauses zum Versorgungsauftrag des MVZ sowie eine Verpflichtung zur Erfüllung des gesamten
Versorgungsauftrages des jeweiligen Fachgebiets vor. ${ }^{31}$ Von der Aufnahme dieser im Vergleich zum Status quo weitergehenden Beschränkungen sah der Gesetzgeber bisher jedoch im humanmedizinischen Bereich ab, sondern hat Einschränkungen von zur Gründung berechtigten Krankenhäusern durch das TSVG nur bei zahnärztlichen MVZ vorgenommen.

Prof. Dr. Peter Wigge

Rechtsanwalt

Fachanwalt für Medizinrecht

Hendrik Hörnlein

Rechtsanwalt
Rechtsanwälte Wigge Scharnhorststraße 40 48151 Münster

Telefon: (0251) 53 595-0

Telefax: (0251) 53 595-99

E-Mail: kanzlei@ra-wigge.de www.ra-wigge.de 